\title{
¿QUÉ CARRERA PARA QUÉ MEDICINA? EL FRACASO DE LA INTRODUCCIÓN DE LAS CIENCIAS SOCIOSANITARIAS EN LA FORMACIÓN MÉDICA DEL PRIMER FRANQUISMO (1938-1959)*
}

\author{
Which Degree for which Profession of Medicine in Spain? The \\ Failure of the Introduction of Social Health Sciences in the Medical \\ Education of the First Franco Regime (1938-1959)
}

\section{Josep Barceló-Prats ${ }^{\alpha}$ y Josep M. Comelles ${ }^{\beta}$}

Fecha de recepción: 0/12/2020 • Fecha de aceptación: 08/03/2021

Resumen. Desde finales del siglo XVIII, la eclosión de una medicina hospitalaria y, medio siglo después, el desarrollo de la medicina experimental y de la estadística sanitaria transformaron la manera de enseñar la medicina. Este nuevo modelo de formación privilegió la «mirada clínica» en detrimento de la mirada sobre el medio social dónde se ejercía la práctica profesional. A pesar de esta subordinación, a escala internacional, la medicina social y las demás ciencias sociosanitarias continuaron siendo materias importantes en el currículum médico. En España, sin embargo, la presencia de ciencias sociosanitarias en las facultades de medicina fue muy escasa. En este artículo, centrado sobre todo en el primer franquismo, pretendemos

\footnotetext{
"Este trabajo se ha realizado en el marco de los proyectos de investigación: «Las claves históricas del desarrollo hospitalario en España y su comparación internacional durante el siglo XX» (RTI2018094676-B-I00) y «Lucha contra el cáncer y cambio sociocultural en España (1939-1975): entre el miedo y la esperanza» (PID2019-107658GB-I00), ambos financiados por el Ministerio de Ciencia e Innovación. Nuestro agradecimiento a Eduardo Bueno, Mercedes del Cura, Josep Danon, Enrique Perdiguero-Gil, Esteban Rodríguez-Ocaña y Alfons Zarzoso. Queremos dedicar este manuscrito a María José Báguena.

a Departamento de Enfermería, Facultad de Enfermería, Universitat Rovira i Virgili. Avinguda de Catalunya, 35, 43002 Tarragona, España. josep.barcelo@urv.cat (D) https://orcid.org/0000-0002-88180872

Departamento de Antropología, Filosofía y Trabajo Social, Facultad de Letras, Universitat Rovira i Virgili. Avinguda de Catalunya, 35, 43002 Tarragona, España. josepmaria.comelles@iubilo.urv.cat (iD) https://orcid.org/0000-0003-0064-364X
}

Cómo citar este artículo: Barceló-Prats, Josep y Comelles, Josep M. «¿Qué carrera para qué medicina? El fracaso de la introducción de las ciencias sociosanitarias en la formación médica del primer franquismo (1938-1959)». Historia y Memoria de la Educación 15 (2022): 29-61 
esclarecer las raíces y las razones de esta marginalidad, tales como el «reglamentismo» legal y administrativo de la universidad española; las resistencias académicas y corporativas a incorporar enfoques interdisciplinares; y un contexto político e ideológico que retrasó, hasta la Transición democrática, la evolución de la sanidad española hacia el concepto de «salud pública». Para ello dividimos el artículo en dos partes. En una primera se repasa la evolución de la formación médica desde mediados del siglo XIX hasta 1938, mientras que en la segunda se describen y analizan las principales reformas de los planes de estudio de medicina realizadas entre 1938 y 1959.

Palabras clave: Formación médica; España; Franquismo; Medicina social; Ciencias sociosanitarias.

Abstract. Beginning at the end of the 18th century, the emergence of hospital medicine and, half a century later, the development of experimental medicine and health statistics, transformed the way medicine was taught. This new training model prioritized the "clinical view", to the detriment of the view on the social environment where professional practice was undertaken. Despite this subordination, on an international scale, social medicine and other social and health sciences continued to be important subjects in the medical curriculum. However, in Spain the presence of social and health sciences was scant. In this article, focused mainly on the early Franco regime, we intend to clarify the roots and reasons for this marginalization, such as the legal and administrative "regulations" of the Spanish university; academic and corporate resistance to incorporating interdisciplinary approaches; and a political and ideological context that delayed, until the Spanish Transition, the evolution of Spanish health towards the concept of "public health". To do this, we have divided the article into two parts. The first follows the evolution of medical training from the middle of the 19th century to 1938, while the second describes and analyses the reforms of medical curricula made between 1938 and 1959.

Keywords: Medical education; Spain; Francoism; Social medicine; Social sciences in medicine.

\section{INTRODUCCIÓN}

La ciencia de conservar la salud y prevenir la enfermedad debería ser enseñada como una de las que poseen mayor importancia. Sería útil para todos y de manera especial para los estudiantes de medicina curativa. ${ }^{1}$

\footnotetext{
${ }^{1}$ Wilson G. Smillie, Medicina preventiva y sanidad pública (Madrid: Espasa-Calpe, 1949), 5.
} 
En 1944, el Ministerio de Educación Nacional publicaba el Decreto de Ordenación de la Facultad de Medicina. En su exposición de motivos se justificaba esta reforma puesto que «al Nuevo Estado cumple vindicar en su plenitud el hondo sentido de la actitud médica, [...] no cabe olvidar que el enfermo ha de ser siempre considerado como un hombre entero, dotado de alma y cuerpo". ${ }^{2}$ Sin embargo, este discurso teórico sobre la misión humanista del médico entró en contradicción con un plan de estudios donde la «medicina social $»^{3}$ y las principales ciencias sociosanitarias —esto es, la sociología y la antropología médicas de raigambre empírica ${ }^{4}$ y no filosófica- ${ }^{5}$ eran marginales. En la España del primer franquismo, por razones ideológicas, políticas y corporativas, ${ }^{6}$ la endeble presencia en la carrera de medicina de las ciencias sociomédicas mencionadas contrastaba con un contexto internacional que ya favorecía su desarrollo incluso antes de la fundación de la Organización Mundial de la Salud (OMS). ${ }^{7}$

\footnotetext{
${ }_{2}$ Decreto de 7 de julio de 1944, sobre la Ordenación de la Facultad de Medicina. BOE de 4 de agosto de 1944, 217, 5943-5950.

${ }^{3}$ En los años treinta del siglo XX, la «medicina social» fue definida como «el arte de prevenir y curar, considerando sus bases científicas, así como sus aplicaciones individuales y colectivas, desde el punto de vista de las relaciones recíprocas que vinculan la salud de los hombres a dichas condiciones». [René Sand, Vers la Médicine Sociale (Paris: Librairie Baillière et Fils, 1948), 576]. Una síntesis de la evolución del concepto, en España, puede verse en: Jesús de Miguel, «Fundamentos de sociología de la medicina», Papers: Revista de Sociología 5 (1976): 209-239. Sobre la implantación y desarrollo de la medicina social en España, véanse Esteban Rodríguez-Ocaña, La constitución de la Medicina Social como disciplina en España, 1882-1923 (Madrid: Ministerio de Sanidad y Consumo, 1987); Esteban Rodríguez-Ocaña, «Ocaso de la medicina social en España: El caso de la Leptospirosis», Asclepio 69, n. 2 (2017): 1-12.
}

${ }^{4}$ Sobre el desarrollo de la sociología y la antropología médicas en España, véanse Joan Campos y Jesús de Miguel, «Sociólogos de bata blanca y médicos con barba: Relaciones entre las ciencias sociales y la Medicina en España», Revista Española Investigaciones Sociológicas 15 (1981): 133-144; Ángel Martínez-Hernáez, Enrique Perdiguero-Gil y Josep M. Comelles, «Genealogía de la Antropología médica en España», Revista de Dialectología y Tradiciones Populares 70 (2015): 205-233.

${ }_{5}^{5}$ Para un análisis del contenido ideológico de las memorias de oposiciones a cátedras de medicina del primer franquismo en las cuales, incluso en materias experimentales, se incluyen disertaciones filosóficas y teológicas, véase José M. López Sánchez, «La Medicina en la universidad nacionalcatólica», en La Universidad Nacionalcatólica. La reacción antimoderna, ed. Luis E. Otero, (Madrid: Universidad Carlos III, 2014), 317-476.

${ }^{6}$ Enrique Perdiguero-Gil, Josep M. Comelles y Aida Terrón, «La introducción de las ciencias sociomédicas en el ámbito de la educación sanitaria y la educación médica durante el franquismo», en $A l$ Servicio de la salud humana. La historia de la medicina ante los retos del Siglo XX, ed. Alfons Zarzoso y Jon Arrizabalaga (Sant Feliu de Guixols: SEHM, 2017), 123-128.

7 Nicoletta Diasio. La science impure. Anthropologie et médecine en France, Grande-Bretagne, Italie, Pays-Bas (Paris: PUF, 1999). 
Paradójicamente, esta realidad chocaba con la que, cuarenta años antes —en 1904—, 8 había conducido a reformar los estudios de medicina para que, mediante la enseñanza de la "higiene», ${ }^{9}$ se impartiesen los contenidos apropiados a los problemas que el médico práctico tenía que resolver en sus relaciones con la sociedad dónde este ejercía. La necesidad de reducir la distancia entre la formación clínica, centrada en el hospital, y la realidad del ejercicio profesional fue una constante en la educación médica internacional desde el siglo XIX. ${ }^{10} \mathrm{~A}$ pesar de esta tensión, en la España de entre siglos existía la voluntad de apostar por la enseñanza de la antropología ${ }^{11}$ y de ampliar los contenidos de higiene en los estudios de medicina -incluso extenderla al Bachillerato y a las Escuelas Normales-. El conocimiento del medio social aún se consideraba indispensable para el correcto desarrollo del ejercicio médico. ${ }^{12}$

En este artículo pretendemos esclarecer las razones que impidieron una mayor presencia de la higiene, la medicina social y otras ciencias sociosanitarias en los planes de estudio de medicina, poniendo el énfasis en el período del franquismo autárquico. Tras un primer apartado donde se analiza la formación médica desde mediados del siglo XIX hasta la Guerra Civil, se describen las reformas efectuadas durante el primer franquismo. Para analizarlas con detalle, dividimos este periodo en dos etapas. Una primera (1938-1951) en la que se debatió la Ley de Ordenación

\footnotetext{
${ }_{8}$ Real Decreto de 10 de junio de 1904, sobre las Cátedras de Higiene con prácticas de Bacteriología sanitaria. Gaceta de Madrid de 12 de junio de 1904, 164, 1013-1014.

${ }^{9} \mathrm{La}$ «higiene» es una parte de la medicina que trata de cuanto «es necesario para conservar la salud, prolongar la vida y mejorar la especie». [Gonzalo Piédrola, Agustín Pumarola y José Bravo, Higiene, medicina preventiva y social (Madrid: Amaro, 1964 [1. ${ }^{\mathrm{a}}$ ed.]), 8]. Durante buena parte del siglo XX «higiene» $\mathrm{y}$ "sanidad» se definían de una manera análoga, pero con un leve matiz separativo "ya que, si hablamos de doctrina pura, de conocimientos teóricos, de lo deseable, usamos la palabra higiene y si esta concepción se liga a una idea de ejecución práctica, preferimos el vocablo sanidad». [Piédrola, Pumarola y Bravo, Higiene (1. ${ }^{\mathrm{a}}$ ed.), 20].

${ }^{10}$ Una síntesis de esta disyuntiva está en Thomas N. Bonner, Becoming a physician. Medical education in Britain, France, Germany, and the United States, 1750-1945 (New York: Oxford University Press, 1995).

${ }^{11}$ Julián Calleja, Necesidad de proteger los estudios antropológicos en nuestro país. Discurso leído ante la Real Academia de Ciencias exactas, físicas y naturales de Madrid (Madrid: Imprenta de L. Aguado, 1892).

12 Medio siglo más tarde, Primitivo de la Quintana ratificaba que, a diferencia de lo acontecido en España, las disciplinas sociomédicas continuaban teniendo relevancia en la formación médica internacional. Primitivo de la Quintana, «La Medicina social como problema docente: evolución conceptual y desarrollo». Revista de Educación 5, n. 13 (1953): 110-123.
} 
Universitaria de 1943 y, con su despliegue, ${ }^{13}$ se reordenaron los estudios de medicina. ${ }^{14}$ Finalmente, una segunda (1951-1959) que estuvo caracterizada por las modificaciones que se introdujeron en los planes de estudios médicos en 1953 y 1959.15

\section{QUÉ LEGISLACIÓN PARA QUÉ PROFESIÓN (1843-1938)}

Desde la creación de la primera escuela médica medieval, en Salerno, la enseñanza de la medicina nunca ha sido una formación más. En el periodo de este epígrafe venía a suponer casi un cuarto de la matrícula universitaria total, solo por detrás de derecho. Ambas profesiones dejaban en una posición residual a las otras facultades. La condición aplicada de la medicina explica que no se pueda abordar la evolución de su formación pre y postgraduada y del Doctorado sin conocer su regulación legislativa, así como la relativa al número, distribución y organización de las universidades y facultades. ${ }^{16}$

En España, por primera vez en 1843,17 a instancias del médico Pedro Mata, se dotaron 22 cátedras en las Facultades de Medicina, una por asignatura, separando la de Higiene de la de Fisiología. La asignatura de Higiene se dividió en Higiene Pública e Higiene Privada y, en ambas, se explicaban las relaciones entre el medio y la enfermedad..$^{18}$ En esa reforma, solo se concedió la categoría de Facultades de Medicina a los Colegios de Cirugía de Madrid y Barcelona, mientras que Santiago, Sevilla,

\footnotetext{
13 Mariano Peset, «La Ley de Ordenación Universitaria de 1943», en Carreras y Ruiz, La universidad española, 125-158.

14 Véase el Decreto de 1944 citado en la nota 2.

15 Decreto de 11 de agosto de 1953, por el que se establecen los planes de estudios de las Facultades de Filosofía y Letras, Ciencias, Derecho, Medicina, Veterinaria y Ciencias Políticas, Económicas y Comerciales. BOE de 29 de agosto de 1953, 241, 5185-5190; Decreto 1742/1959, de 23 de septiembre, por el que se reduce el plan de estudios del periodo de Licenciatura de la Facultad de Medicina. BOE de 12 de octubre de 1959, 244, 13132.

${ }_{16}$ Sobre el origen de diversas Facultades de Medicina, véase José Danón (coord.), La enseñanza de la Medicina en la universidad española: primera parte (Barcelona: Fundación Uriach, 1998). Un segundo volúmen del libro, con idéntico título, se publicó en 2001.

${ }_{17}$ Sobre la enseñanza de la medicina antes de 1843, véase: Mariano Peset, José L. Peset, La universidad española (siglos XVIII y XIX): despotismo ilustrado y revolución liberal (Madrid: Taurus, 1974).

18 Pedro F. Monlau. Elementos de higiene pública o el arte de conservar la salud de los pueblos (Madrid: Imprenta de M. Rivadeneyra, 1862, 3 vols.); Pedro F. Monlau. Elementos de higiene privada o el arte de conservar la salud del individuo (Madrid: Librería de Moya y Plaza, 1864).
} 
Valencia, Valladolid y Zaragoza quedaron reducidas a «colegios de prácticos en el arte de curar destinados a formar titulados de segunda clase». ${ }^{19}$ La reforma universitaria del ministro Pidal amplió, en 1845, la formación de médicos a Cádiz, Santiago y Valencia. Esta estructura, tras algunos retoques en 1847 y 1849, quedó fijada definitivamente durante más de un siglo con la Ley Moyano de 1857.20

En lo sucesivo, en medicina, la Higiene privada y pública se impartían en séptimo curso y en el Doctorado. ${ }^{21}$ Según Monlau, esta "proclamación legal de la importancia de los estudios antropológicos, fue la iniciación práctica de la Medicina en la buena Administración pública». ${ }^{22}$ La formación médica combinaba, por entonces, conocimiento y práctica clínica con la necesidad de comprender la influencia del medio -entendido en un sentido amplio- en la etiología de la enfermedad. ${ }^{23}$ Esto último quedaba incluido en la Higiene, siendo una materia fundamental para desarrollar una práctica profesional atenta a los campos considerados como medicina social. ${ }^{24}$ Tanto es así que las topografías y geografías médicas, ${ }^{25}$ los informes médico-sociales y la publicación de otros textos de índole sociosanitaria avalaron su valor para el quehacer profesional, junto a los conocimientos de epidemiología, estadística, sociología o etnografía. ${ }^{26}$ Por eso, médicos como Pedro Felipe Monlau eran

19 José M. López-Piñero, «La enseñanza médica en España desde la Baja Edad Media hasta la Ley Moyano (1857)», en Danón, La enseñanza de la Medicina, 7-29, 26.

${ }^{20}$ López-Piñero, «La enseñanza médica en España», 27.

21 Sobre la evolución de la titulación médica hasta la Segunda República, véase Alejandro Ridruejo, «La enseñanza de la medicina en España: planes de estudio (1843-1931)» (Tesis doctoral: Universidad de Valladolid, 1979).

${ }^{22} \mathrm{M}^{\mathrm{a}}$ José Báguena, «La higiene y la salud pública en el marco universitario español», Revista de Sanidad e Higiene Pública 68 (1994): 94.

23 Sobre las relaciones entre enfermedad y medio, véanse: Philippe Pinel, Nosografía filosófica o Aplicación del método analítico á la medicina (Madrid: Imprenta Real, 1803); Leonhard L. Finke, Versuch einer allgemeinen medicinisch-praktischen Geographie, worin der historische Theil der einheimischen Völker- und Staaten-Arzeneykunde vorgetragen wird, (Leipzig: in der Weidmannschen Buchhandlung, 1795).

${ }^{24}$ George Rosen, De la policía médica a la medicina social: ensayos sobre la historia de la atención a la salud (Mexico: Siglo XXI, 1985).

25 Véase, por ejemplo: Juan Casco-Solís, «Las topografias médicas: Revisión y cronologia», Asclepio 53, n. ${ }^{\circ} 1$ (2001): 213-244.

26 Josep M. Comelles, «The Role of Local Knowledge in Medical Practice: A Trans-Historical Perspective», Culture, Medicine and Psychiatry 24, n. ${ }^{\circ}$ (2000): 41-75. 
críticos con una formación exclusivamente hospitalaria y clínica que no habilitaba para ejercer en contextos geográficos y culturales diversos. ${ }^{27}$ Este último insistía en:

que los discípulos sean conducidos [...] a las grandes fábricas, a los hospitales y a las cárceles, a los buques y a los cuarteles, para estudiar las influencias patogénicas, para enterarse de los procederes manuales de las artes, para examinar las condiciones atmosferológicas, etc. [...] Durante el curso se le debe acompañar a estudiar prácticamente el laboreo de minas, la policía rural, y ensayarle en la formación de topografías. ${ }^{28}$

Inicialmente, solo se incluyeron conocimientos sobre el medio en la asignatura de Higiene privada y pública, ${ }^{29}$ pero el plan de 1884 añadió, en sexto curso de la Licenciatura, Nociones de Estadística Médica a la Higiene Pública. Fue en el Doctorado, centralizado en la Universidad de Madrid, donde se introdujeron Filosofía Médica, Historia Crítica de la Medicina General y particularmente en España, así como Estudio Histórico y Geográfico de las Principales Epidemias y Particularmente de las Ocurridas en España. ${ }^{30}$ En 1886, el plan Montero eliminó Filosofía Médica, mantuvo Historia Crítica de la Medicina y sustituyó el Estudio Histórico de las Epidemias por una ampliación de la Higiene Pública y Epidemiología. Las limitaciones de los conocimientos sociosanitarios en la Licenciatura explican que, en 1892, el anatomista Julián Calleja vindicase la inclusión en ella de la Antropología. ${ }^{31}$ Esa disciplina incluía, entonces, Antropología Física, Etnología — ambas con trascendencia en medicina práctica一, Filología y Arqueología. Sin embargo, la propuesta

\footnotetext{
27 Josep M. Comelles, «From Ethnography to Clinical Practice in the Construction of the Contemporary State», en Democracy and Ethnography: Constructing Identities in Multicultural Liberal States, ed. Carol J. Greenhouse (Albany: State University of New York Press, 1998), 233-253.

${ }^{28}$ Báguena, «La higiene», 95.

${ }^{29}$ En 1850, el plan Seijas redujo la Higiene Privada a nociones muy generales de fisiología. En 1852 su docencia volvió a manos del catedrático de Higiene. El plan Moyano desdobló la Higiene en «Privada» y «Pública», reunificándose, en 1867, como Higiene Pública y Privada y creándose, para el Doctorado, unos estudios superiores de Higiene Pública y Epidemiología.

${ }^{30}$ Ridruejo, La Enseñanza de la Medicina, 384. Nótese que la última materia es la traducción del título de August Hirsch, Handbuch der Historisch-Geographischen Pathologie (Erlangen: Verlag von Ferdinand Enke, 1860).

${ }^{31}$ Calleja, Necesidad de proteger.
} 
de Calleja solo se aplicó a las Facultades de Ciencias y no a la de Medicina.

En 1905, se promulgó el temario oficial de las oposiciones a médicos titulares. ${ }^{32}$ Estaba dividido en cinco bloques, cuatro de temario clínico y un quinto, con 26 temas, reservado a la higiene. Sin embargo, no se aprecia el menor atisbo de la sociología o la antropología en ninguno de esos temas, puesto que, al poder acceder a dichas oposiciones cualquier licenciado sin otro requisito, el temario había de ajustarse al contenido de la carrera.

El escaso peso de las ciencias sociosanitarias en la Licenciatura, desde 1904,33 explica el despliegue, una vez creada la Escuela Nacional de Sanidad (ENS), ${ }^{34}$ de una oferta de cursos de postgrado con contendidos en medicina social destinados a los nuevos especialistas de la sanidad pública. $^{35}$

La reforma del plan de 1928, posterior a la creación de la mencionada ENS, reforzó la formación clínica del médico general —con conocimientos básicos de algunas especialidades- en detrimento de las ciencias sociomédicas. En un momento histórico en que buena parte de la práctica médica aún continuaba ejerciéndose sobre obreros y campesinos, ${ }^{36}$ los contenidos de medicina social se limitaron a una sola asignatura de Higiene, en tercer curso, y a Pediatría, en sexto.

En este periodo, a escala internacional, ya se había producido la eclosión disciplinar de la sociología empírica y de la antropología social

\footnotetext{
32 Real Orden de 23 de marzo de 1905, sobre el Programa de oposiciones a ingreso en el Cuerpo de Médicos titulares. Gaceta de Madrid de 24 de marzo de 1905, 83, 1124-1125.

${ }_{33}$ El Real Decreto de 1904, citado en la nota 8, refundió las cátedras de Higiene en una sola, impartiéndose esa única asignatura en sexto curso de la Licenciatura.

${ }^{34}$ Gustavo Pittaluga, La constitución de la Escuela Nacional de Sanidad de Madrid (Madrid: Publicaciones de la Escuela Nacional de Sanidad, 1930). Desde 1920 también había jugado un papel importante en el desarrollo de la salud pública la Fundación Rockefeller. Esteban Rodríguez-Ocaña, «La intervención de la Fundación Rockefeller en la creación de la sanidad contemporánea en España», Revista Española de Salud Pública 74 (2000): 27-34.

35 Jorge Molero e Isabel Jiménez, «Salud y burocracia en España. Los Cuerpos de Sanidad Nacional, 1855-1951», Revista Española de Salud Pública 74 (2000): 45-79.

${ }_{36}$ Así se detalla, por ejemplo, en Francisco Polo Fiayo, El gran esclavo, el médico (Madrid: Jesús Morata, 1929); Juan M. Lara. De la vida médica rural (Dos Hermanas: Imprenta Diez, 1945).
} 
y cultural. ${ }^{37}$ En un principio, ambas quedaron alejadas de la medicina por el énfasis científico-experimental de esta última. ${ }^{38}$ Esto es, algunos de los rasgos constitutivos del llamado "modelo médico", ${ }^{39}$ tales como su asocialidad, ahistoricidad e individualismo metodológico, privilegiaron la mirada clínica respecto de la efectuada sobre el medio social. Ello dio lugar a novedades didácticas como la lección clínica o el internado de los estudiantes en los hospitales, así como la construcción de un "paciente hospitalario». ${ }^{40}$ Así, inicialmente, los intereses comunes entre la medicina y la antropología quedaron reducidos a temas ubicados en los márgenes de ambas disciplinas, ${ }^{41}$ como el conocimiento de la diversidad local de las enfermedades, el folklore o los debates con las escuelas psicoanalíticas. ${ }^{42}$ Con la sociología pueden documentarse algunas relaciones más, sobre todo en temas de organización profesional y hospitalaria, en higiene y sanidad, en medicina social y en torno a las problemáticas vinculadas con el desarrollo de los seguros médicos. ${ }^{43}$

En España, antes de 1936, se documentan traducciones de libros de sociología y de antropología. ${ }^{44}$ Sin embargo, las relaciones entre la medicina y estas disciplinas fueron casi nulas. Esta realidad no varió

\footnotetext{
37 George W. Stocking, «The Ethnographic Sensibility of the 1920s and the Dualism of the Anthropological Tradition», en Romantic Motives. Essays on Anthropological Sensibility, ed. George W. Stocking (Madison: The University of Wisconsin Press, 1989), 208-276.

${ }_{38}$ Sobre el nacimiento de la clínica, véase Michel Foucault, El nacimiento de la clínica: una arqueología de la mirada médica (México: Siglo XXI editores, 2001). Sobre la introducción de las ciencias básicas y el peso del laboratorio en la formación médica, véase Thomas Neville, Becoming a physician. Medical education in Britain, France, Germany, and the United States, 1750-1945 (Oxford: Oxford University Press, 1995).
}

${ }^{39}$ Eduardo L. Menéndez, «El Modelo Médico y la Salud de los Trabajadores», Salud Colectiva 1, n. 1 (2005): 9-32.

${ }^{40}$ Colin Jones, «The construction of the hospital patient in Early Modern France», en Institutions of Confinement. Hospitals, Asylums and Prisions in Western Europe and North America, ed. Norbert Finzsch y Robert Jütte (Cambridge: Cambridge University Press, 1996), 55-74.

${ }_{41}$ Josep M. Comelles y Ángel Martínez-Hernáez, Enfermedad, cultura y sociedad. Un ensayo sobre las relaciones entre la Antropología social y la Medicina (Madrid: EUDEMA, 1993).

42 George W. Stocking, «Anthropology and the Science of the Irrational. Malinoswski's Encounter with Freudian Psychoanalysis», en Malinowski, Rivers, Benedict and Others. Essays on Culture and Personality, ed. George W. Stocking (Madison: The University of Wisconsin Press, 1986), 13-49.

43 Patricia Kendall y George G. Reader, «Contributions of Sociology to Medicine», en Handbook of Medical Sociology, ed. Howard E. Freeman, Sol Levine y Leo G. Reeder (New Jersey: Prentice Hall, 1972), 1-29.

${ }_{44}$ Como, por ejemplo, la traducción de la obra de Émile Durkheim, El suicidio (Madrid: Editorial Reus, 1927); o la de Bronislaw Malinowski, La vida sexual de los salvajes del Noroeste de Melanesia (Madrid: Morata, 1932). 
con la Segunda República, ${ }^{45}$ puesto que los contenidos sociosanitarios en la carrera de medicina continuaron impartiéndose casi exclusivamente en Higiene, una cátedra que, además, solían ocupar provisionalmente profesores interinos de paso hacia puestos más codiciados. ${ }^{46}$ Tras la Guerra Civil, como veremos, la situación no cambió en exceso. Por eso, en 1953, se continuaba alertando que:

Lo que no es tolerable es la graduación para el ejercicio de una profesión determinada sin haber incluido en el currículum las enseñanzas necesarias de disciplinas fundamentales o formativas que pueden [...] suponer un riesgo de fracaso en el global de la enseñanza. [...] Creemos que en este último caso se encuentra la enseñanza de la medicina social. ${ }^{47}$

\section{QUÉ CARRERA PARA QUÉ MÉDICOS (1938-1951)}

\section{La Universidad en el Nuevo Estado}

En 1938, un joven psiquiatra, Juan José López Ibor escribía, desde la radicalidad ideológica del falangismo, que «España no ha tenido Universidad auténtica, en los últimos tiempos, porque no podía tenerla».48 Afirmaba que el Nuevo Estado podía hacerla posible. Ello se intentó, en 1943, con la Ley de Ordenación Universitaria (LOU). ${ }^{49}$ Por un lado, el exilio y las depuraciones dejaron numerosas cátedras vacantes que fueron ocupadas por afines al régimen..$^{50}$ Para muchos de sus titulares era

\footnotetext{
${ }_{45}$ Sobre la tarea sanitaria de la Segunda República, véanse Joan Serrallonga, «Reformadores y reaccionarios en la estructura central de sanidad en España, 1931-1936», Investigaciones Históricas 29 (2009): 241-264; Ferrán Martínez-Navarro, «La Sanidad en España», Revista de Sanidad e Higiene Pública 51 (1977): 777-817.

46 Báguena, «La higiene», 96.

${ }_{47}$ Primitivo de la Quintana, «La Medicina social como problema docente», Revista de Educación 5, n. 12 (1953): 1-11, 1.

48 Juan J. López Ibor, Discurso a los universitarios españoles (Santander: Cultura española, 1938). Reeditado (Madrid: Rialp, 1957), 109.

49 Alicia Alted-Vigil, «Bases político-ideológicas y jurídicas de la universidad franquista durante los Ministerios de Sainz Rodríguez y primera época de Ibáñez Martín (1938-1945)», en Carreras y Ruiz, La universidad española, 95-124, 97.

${ }^{50}$ Alfons Zarzoso y Àlvar Martínez-Vidal, Medicina, guerra y exili. Una generació destruïda per la guerra (Barcelona: CSIC, 2011).
} 
una prebenda que les permitía acceder a negocios privados y formar parte de la "élite cultural» ${ }^{51}$ del régimen o mantener la condición de notables locales como antes de la Guerra Civil. Por otro lado, la LOU no amplió el número universidades ni el de cátedras, lo que dio lugar a agrupar — a menudo arbitrariamente - disciplinas consideradas afines a las cátedras existentes. Esto dificultó que disciplinas como la sociología y la antropología pudieran ser transversales en distintas facultades, siendo ubicadas, principalmente, en las Facultades de Filosofía y Letras, ${ }^{52}$ de Derecho y, más tarde, de Economía.

En el debate ideológico sobre la LOU se confrontaron partidarios de una «universidad científica» y quiénes, influidos por Ortega y Gasset, postulaban por una «universidad cultural». ${ }^{53}$ López Ibor, médico, no creía en la posibilidad de ensamblar los estudios de medicina con los ubicados en Letras, «ya que no es posible hacerle seguir a un médico [...] varios cursos en una Facultad de Cultura en detrimento de su formación médica»,54 Pero, al mismo tiempo, consideraba que un profesional solo «embebido de ciencia y de técnica [...] no es más que una traducción de la idea luterana de profesión que nunca encajó en nuestro modo de ser».55

Tras un intento fallido de Ley de Universidades de 1939,56 se redactó el anteproyecto de la Ley de 1943, apuntando ideas populistas como la universidad gratuita. ${ }^{57}$ Sin embargo, la presión del nacionalcatolicismo influyó en la redacción final, más conservadora, ${ }^{58}$ que se terminó promulgando sin demasiada oposición del ministro Ibáñez Martín. ${ }^{59}$ Dicha

\footnotetext{
51 Sobre el concepto de «élite cultural», véase: Nicolás Sesma, «Sociología del Instituto de Estudios Políticos. Un "grupo de élite" intelectual al servicio del Partido Único y del Estado franquista», en Falange: las culturas políticas del fascismo en la España de Franco (1936-1975), ed. Miguel Á. Ruiz Carnicer (Zaragoza: Institución Fernando el Católico, 2013), 253-288.

52 Decreto de 7 de Julio de 1944, sobre la ordenación de la Facultad de Filosofía y Letras. BOE de 4 de agosto de 1944, 217, 5912-5926.

53 Luis E. Otero, «La universidad nacionalcatólica», en Otero, La Universidad Nacionalcatólica, 69-130.

${ }^{54}$ López Ibor, Discurso a los universitarios, 64-65.

55 López Ibor, Discurso a los universitarios, 66.

56 Otero, «La universidad nacionalcatólica», 91-93.

57 Peset, «La Ley de Ordenación», 126.

58 Pedro Laín Entralgo, Descargo de Conciencia, 1930-1960 (Barcelona: Barral, 1976), 292-293.

59 Alted-Vigil, «Bases político-ideológicas», 98-99.
} 
ley limitó al extremo la autonomía de las universidades, regulando minuciosamente el acceso a los puestos docentes y a los órganos de gobierno para asegurar su control ideológico y político. ${ }^{60}$ Este férreo control explica la imposibilidad de reformar a fondo la legislación universitaria hasta la Ley General de Educación de 1970 y, sobre todo, la Ley de Reforma Universitaria de 1984.

\section{¿Una nueva carrera de Medicina?}

En 1944, un Decreto regularizó todas las facultades y en la de Medicina se abordó «el arduo problema de la especialización. [...] El caso más general era siempre el de un autodidactismo de los especialistas, cuya formación se realizaba fuera de las facultades y conducía frecuentemente a dolorosos resultados».61 Este Decreto abrió las puertas a la utopía de una medicina autárquica al margen, por razones ideológicas, de la ciencia experimental de su tiempo.62 Sin embargo, ese mismo Decreto creaba el título de especialista, una novedad ajustada a la evolución médica y que ya se incorporó, ese mismo año, a la planificación del nuevo Seguro Obligatorio de Enfermedad (SOE). ${ }^{63}$ Se amplió el Doctorado a todas las facultades y, para elevar su calidad, se establecieron criterios rigurosos y selectivos para formar investigadores. Las enseñanzas del grado de Doctor en Medicina consistían en «cuatro cursos monográficos que podrán versar, entre otras materias, sobre Matemáticas para Biólogos, Antropología -física-, Hidrología médica, Medicina colonial y Psicología experimental y racional. En vez de uno de los cursos monográficos el alumno podrá cursar una disciplina de cualquier otra Facultad».64

\footnotetext{
60 Mariano Peset, «Prólogo», en Oposiciones y concursos a cátedras de Historia en la Universidad de Franco (1939-1950), ed. Yolanda Blasco y María F. Mancebo (València: Publicacions de la Universitat de València, 2010), 11-22.

${ }^{61}$ Decreto de 7 de julio de 1944, sobre la Ordenación de la Facultad de Medicina. BOE de 4 de agosto de $1944,217,5944$.

62 Sobre los efectos del Decreto en la ideología y el perfil del profesorado que se incorporó durante el primer franquismo, véase López Sánchez, «La Medicina en la universidad», 317-320.

${ }_{63}$ Primitivo de la Quintana, «Plan de asistencia médica y medicina preventiva para la implantación del seguro de enfermedad en España», Profesión Médica 20, (1944): 1-4.

${ }^{64}$ Decreto de 7 de julio de 1944, sobre la Ordenación de la Facultad de Medicina. BOE de 4 de agosto de $1944,217,5948$.
} 
Por su parte, la Licenciatura constaba de una etapa preclínica, donde se buscaba dar al estudiante una formación de tipo general y una clínica que pretendía alcanzar la completa capacidad profesional mediante las prácticas hospitalarias. La docencia combinaba lecciones de cátedra con el pase de visita por las salas de los hospitales del profesor con los estudiantes. Asimismo, existía la posibilidad de que los estudiantes se matriculasen como libres, eso es, con derecho a examen, pero sin obligación de asistir a las clases. Los exámenes consistían en memorizar teoría y exponerla de forma oral o escrita. Por eso, el alumnado, en particular el libre, utilizaba manuales, apuntes de cátedra o lecciones para estudiar el temario de las diversas asignaturas.

En un contexto laboral aún de escasa especialización, la Licenciatura siguió habilitando para un ejercicio cuyas principales salidas profesionales continuaban siendo las oposiciones a médico titular de Asistencia Pública Domiciliaria (APD) ${ }^{65}$ o a Cuerpos de Sanidad y a ejercer en el SOE como médico militar o en el ámbito privado.

\section{De apuntes y manuales con contenidos de medicina social}

Los manuales existentes para estudiar las asignaturas que se cursaban en medicina son útiles para documentar dónde se podían impartir la medicina social y las demás ciencias sociosanitarias. Como veremos a continuación, dichos contenidos podían tener cierta cabida en Higiene y Sanidad, Pediatría y Puericultura, Psiquiatría, Psicología e Historia de la Medicina, siendo su distribución y su carga docente muy diversas. Sin embargo, el análisis de las memorias de oposiciones a cátedras de todas estas asignaturas pone de relieve que los candidatos exponían sobre todo argumentos filosóficos e incluso teológicos ajenos a cualquier tipo de empirismo y en ocasiones de experimentalismo. ${ }^{66}$

\footnotetext{
${ }^{65}$ Sobre los médicos titulares, véase Josep M. Comelles, Enrique Perdiguero-Gil, Eduardo Bueno, y Josep Barceló-Prats, «Por caminos y veredas: La práctica médica rural bajo el Franquismo (19391979)», en Genealogías de la Reforma Sanitaria en España, eds. José Martínez-Pérez, Enrique Perdiguero-Gil (Madrid: La Catarata, 2020), 63-124, 77-79.

${ }^{66}$ López Sánchez, «La Medicina en la universidad», 320-375.
} 
A Higiene y Sanidad, asignatura de sexto curso, se le asignaron entre 60 y 80 horas de lecciones dependiendo del calendario. Los contenidos de los diferentes manuales de higiene, publicados entre la década de los cuarenta y la de los sesenta, son muy similares. Esta homogeneidad puede explicarse porque una parte importante de los temarios de algunas oposiciones, como las que daban acceso a los Cuerpos de Sanidad Nacional, versaban sobre contenidos de higiene y medicina preventiva ${ }^{67}$ Los dos primeros manuales del periodo franquista fueron la Higiene Rural ${ }^{68}$ y las Lecciones del curso de Higiene ${ }^{69}$ del catedrático de la Universidad de Valladolid, Emilio Zapatero. En el prólogo del primero, el director general de Sanidad - José Alberto Palanca-, confirma que hasta entonces en la asignatura de Higiene no se había hablado «ni de organización sanitaria, [...] ni de epidemiología, ni de estadística, ni de ingeniería sanitaria, ni de ninguno de los aspectos que hoy día son consubstanciales con la higiene». ${ }^{70}$ Sin embargo, estas temáticas continúan estando poco representadas en ambos manuales.

En los múltiples manuales de Pediatría y Puericultura del periodo, los contenidos sociosanitarios tampoco ocupan un lugar central. ${ }^{71} \mathrm{No}$ solo iban destinados a estudiantes, sino también a médicos en ejercicio y a visitadoras sanitarias, entre otros, puesto que también servían para preparar temarios de exámenes, oposiciones y concursos. Responden al interés compartido por la Dirección General de Sanidad (DGS) y los falangistas de luchar contra la mortalidad infantil, ${ }^{72}$ reflejándose en las propuestas de organización del dispositivo asistencial a la infancia presentes en las colecciones de Al servicio de España y al niño español (DGS) o en el proyecto falangista de Juan L. Morales. ${ }^{73}$

\footnotetext{
${ }^{67}$ Molero y Jiménez, «Salud y burocracia», 72-74.

68 Emilio Zapatero, Higiene Rural (Barcelona: Salvat Editores, 1943).

${ }^{69}$ Emilio Zapatero, Lecciones del curso de Higiene (Valladolid: Facultad de Medicina, 1944).

70 Zapatero, Higiene Rural, 11.

71 Luis Torres, Manual de Pediatría (Barcelona, Bosch, 1940); José Boix, Manual de Puericultura (Valencia: Librerías de García-Muñoz, 1941).

72 Campos y de Miguel, «Sociólogos de bata blanca», 133-141.

73 Juan L. Morales, "Programa totalitario de sanidad y asistencia social infantil», Revista de Sanidad e Higiene Pública 15 (1941): 304-348.
} 
En los manuales de Psiquiatría, el discurso dominante oscilaba entre el eclecticismo y la fenomenología ${ }^{74}$ tomando distancias con las escuelas psicoanalíticas. ${ }^{75} \mathrm{El}$ rastreo de la presencia de la sociología o la antropología es más difícil, ya que los principales psiquiatras del franquismo, como López Ibor, ${ }^{76}$ no tuvieron excesivo interés por los debates internacionales entre ambas disciplinas y las escuelas psicológicas, psicoanalíticas y psiquiátricas. ${ }^{77} \mathrm{El}$ primer manual de psiquiatría, publicado en 1941 por José Pérez López-Villamil, ${ }^{78}$ quiso llenar un vacío que, según su autor, no suplían las explicaciones de cátedra ni la literatura nacional, describiendo la psicopatología general, distanciándose del «organicismo» y explicando su etiología mediante el «constitucionalismo» de Kretschmer. ${ }^{79}$

El decreto de 1944 asignaba Psicología, en segundo curso de la carrera de medicina, a la Facultad de Filosofía y Letras. ${ }^{80}$ Esta situación duró poco, puesto que probablemente sus contenidos, basados allí en una psicología neoescolástica ajena a las ciencias sociales, ${ }^{81}$ no tenían interés para futuros médicos. A finales de la década, la ya denominada Psicología médica se incorporó a las cátedras de Psiquiatría. Los textos más recomendados en esta asignatura fueron los de Ernst Kretschmer. ${ }^{82}$

\footnotetext{
${ }^{74}$ Ricardo Campos y Ángel González, «Psiquiatría en el primer franquismo: saberes y prácticas para un "Nuevo Estado"», Dynamis 37, n.o 1 (2017): 13-21

75 Silvia Levy, Psicoanálisis y defensa social en España, 1923-1959, (Madrid: Los Libros de la Catarata, 2019), 126-153.

76 Juan J. López Ibor, La Agonía Del Psicoanálisis (Madrid: Espasa-Calpe, 1951).

77 Ricardo Campos y Enric Novella, «La Higiene Mental durante el primer franquismo. De la higiene racial a la prevención de la enfermedad mental (1939-1960)», Dynamis 37, n. 1 (2017), 65-87.

78 José Pérez López-Villamil, Manual de Psiquiatria. Psicopatología General (Lugo: Asociación Gallega de Psiquiatria, 2003). Es la reedición del libro de 1941 donde toma distancias de las orientaciones organicistas y aplaude la labor de psiquiatras como Juan J. López Ibor, Emili Mira o Ramón Sarró.

79 Ernst Kretschmer, Constitución y Carácter, (Barcelona-Madrid: Labor, 1954); Ernst Kretschmer, Psicología Médica, (Barcelona-Madrid: Labor, 1954).

${ }^{80}$ Decreto de 7 de julio de 1944 sobre la Ordenación de la Facultad de Filosofía y Letras. BOE de 4 de agosto de 1944, 217, 5912-5926.

81 Levy, Psicoanálisis, 126 y siguientes.

82 Josep Solé, «La Influencia de Kretschmer en la medicina, la psiquiatría y la literatura psicológica española», Revista de Psicología General y Aplicada 4, n. 2 (1949): 475-511.
} 
Finalmente, a Historia de la Medicina, en séptimo curso, se le destinaba una única hora semanal. En esta materia hay que destacar la contribución de Pedro Laín Entralgo. ${ }^{83}$ Primer catedrático de la disciplina cuando esta aún se limitaba al doctorado, Laín tuvo mucha relevancia cultural y política por su posición como uno de los intelectuales orgánicos del franquismo. Ello le llevó a Rector de la Universidad de Madrid entre 1950 y 1956. La Historia de la Medicina, en el último curso, abrió las puertas al futuro desarrollo de la historiografía médica y a la apertura hacia las ciencias sociales. ${ }^{84}$ Sin embargo, la antropología médica que defendía Laín guardaba diferencias sustanciales con los preceptos de la sociología empírica o de la antropología social. ${ }^{85}$ Aunque en su obra cita a algunos de los referentes fundamentales de ambas disciplinas, abogaba por un concepto de antropología médica que, vinculado a la fenomenología y a la filosofía de Xavier Zubiri, ${ }^{86}$ Binswanger y Von Weizsäcker, ${ }^{87}$ examinaba la condición del paciente y su relación con el médico. ${ }^{88}$ Su postura alimentó la necesidad de las humanidades médicas, menos por su peso en la carrera de medicina como por su papel en la construcción de la identidad profesional del médico en un contexto de progresiva socialización de la sanidad española. ${ }^{89}$

\section{Los límites de la medicina social en el primer franquismo}

El Decreto de 1944 abrió las puertas a la formación postgraduada y continuada de los profesionales, no únicamente médicos. En ambas

\footnotetext{
${ }^{83}$ López Sánchez, «La Medicina en la universidad», 382-387.

${ }^{84}$ José M. López-Piñero, «Historia social, antropología cultural y sociología de la medicina en la enseñanza médica. Hacia una ciencia socio-médica», Medicina e Historia 3 (1971): 2-16.

${ }^{85}$ Pedro Laín Entralgo, Estudios de Historia de la Medicina y de Antropologia médica (Madrid: Escorial, 1943).

86 Ángel González de Pablo, «Por la psicopatología hacia Dios: Psiquiatría y saber de salvación durante el primer franquismo", Dynamis 37, n. ${ }^{\circ} 1$ (2017): 45-64.

${ }^{87}$ Pedro Laín Entralgo, El Estado de Enfermedad (Madrid: Sociedad de estudios y publicaciones, 1968).

${ }^{88}$ Pedro Laín Entralgo, La relación médico enfermo: historia y teoría (Madrid: Revista de Occidente, 1964).

${ }^{89}$ Perdiguero-Gil, Enrique y Eduardo Bueno Vergara, «"Hay una diferencia entre la medicina social y la socializada". Las resistencias de los médicos españoles a la colectivización de la asistencia sanitaria y la ampliación de la cobertura sanitaria (1944-1963)», en El Estado Del Bienestar Entre El Franquismo y La Transición, eds. Damián González Madrid y Manuel Ortiz Heras (Madrid: Sílex Editorial, 2020), 95-124.
} 
formaciones puede documentarse el papel de la DGS, de la ENS, ${ }^{90}$ del SOE y de la Delegación Nacional de la Falange Española Tradicionalista y de las Juntas de Ofensiva Nacional Sindicalista (FET y de las JONS), así como de su Sección Femenina. Sin embargo, existía una pugna entre dos concepciones distintas de medicina social. Por un lado, según la retórica falangista, medicina social era sinónimo de socialización de la medicina y se usaba para describir la asistencia del SOE. En la revista $S E R,{ }^{91}$ un órgano falangista, se documentan las diferencias entre la DGS y el SOE, puesto que este último postergaba la salud pública en favor de la atención clínica a la enfermedad. ${ }^{92}$ Por otro lado, la DGS y la ENS, que mantuvieron el concepto clásico de medicina social, se centraron en la realización, entre otras acciones, de campañas de prevención, de formación continuada y de edición de colecciones como Al servicio de España y del Niño Español, ${ }^{93}$ los Folletos de divulgación o la Revista de Sanidad e Higiene Pública.

En los años cuarenta las salidas profesionales del limitado número de nuevos licenciados no habían cambiado. Aunque, en 1944, la aparición del SOE modificó el mercado laboral, la demanda de estudios de medicina continuó relativamente estable hasta los años sesenta. En la medida que la reforma hospitalaria no empezó a discutirse hasta finales de los cincuenta, la principal salida profesional continuó siendo la medicina general. Por su parte, la pediatría y la tocoginecología experimentaron un lento crecimiento, mientras que la cirugía general y las especialidades fueron sectores minoritarios hasta principios de los sesenta. La estabilidad del currículum médico, que apenas matizarían las reformas de 1953 y 1959, con la ampliación de asignaturas de especialidades, tampoco favoreció grandes cambios en una formación que continuó siendo eminentemente clínica.

\footnotetext{
${ }_{90}$ Fernando Ruiz Falcó, «Escuela Nacional de Sanidad. Problemática pasada, actual y futura», Revista de Sanidad e Higiene Pública 57 (1983): 359-372.

${ }^{91}$ Enrique Perdiguero-Gil, "La "educación sanitaria" en el ideario médico-social del franquismo a través de la revista SER», en Política, salud y enfermedad en España: entre el desarrollismo y la transición democrática, ed. Enrique Perdiguero-Gil (Elche: Universidad Miguel Hernández, 2015).

92 En la revista $S E R$ también se documentan comentarios explícitos sobre los nuevos aires formativos internacionales. Véase la sección «Salud Internacional» de Gerardo Clavero.

93 Modesta Salazar, Josep Bernabeu-Mestre y Emilio A. Martínez-Marco, «La salud materno-infantil durante el franquismo: notas bibliométricas sobre el programa "Al Servicio de España y del niño español"», Asclepio 59, n. 1 (2007): 285-314.
} 
Sin embargo, en 1951, el director general de Sanidad —José Alberto Palanca-94 advertía que el modelo de medicina estaba cambiando velozmente por el despliegue del SOE y por el nuevo desarrollo de las especialidades médicas y quirúrgicas. Algunos técnicos de la ENS eran conscientes que esta evolución profesional tendría efectos colaterales por la desconexión de los contenidos reflejados en los manuales aludidos y las nuevas tendencias internacionales en salud pública y educación sanitaria. Quizás por eso, unos años antes —en 1948-, un editorial sobre «educación sanitaria popular»,95 refiriéndose a España y citando fuentes internacionales, consideraba que «uno de los problemas de solución urgente e inaplazable es el de la deficiencia de la educación sanitaria popular». ${ }^{96}$ Describiendo lo conseguido y lamentando la falta de coordinación, añadía que:

Todos los sanitarios conocemos la urgencia de que el público profano, como sujeto de prevención, colabore en su propio beneficio. Hace tiempo que se viene clamando por la enseñanza universal de la higiene en las escuelas, en los cuarteles, en las universidades, [...] pero no se trata solo de higiene en el sentido clásico, sino de una higiene con ribetes de sanidad y sociología. ${ }^{97}$

El mencionado editorial, pues, apostaba porque el conjunto del dispositivo educacional tuviese una formación sanitaria que reconociese el valor de la sociología y la antropología. ${ }^{98}$

Un año más tarde, se traducía el libro Preventive Medicine and Public Health. ${ }^{99} \mathrm{Su}$ autor, el salubrista Wilson M. Smillie, consideraba a la

\footnotetext{
${ }_{94}$ José A. Palanca, «Sobre el personal sanitario y sus problemas y necesidades», Horizonte 3, n. ${ }^{\circ} 18$ 19 (1951): $12-14$

95 Sin autor, «Editorial: La educación sanitaria popular», Revista de Sanidad e Higiene Pública 22, n. 3 (1948): 193-198.

96 Sin autor, «Editorial: La educación», 193.

${ }_{97}$ Sin autor, «Editorial: La educación», 194.

${ }_{98}$ Aida Terrón, Josep M. Comelles y Enrique Perdiguero-Gil, «Schools and Health Education in Spain during the Dictatorship of General Franco (1939-1975)», History of Education Review 46, n. 2 (2017): 208-223.

99 Nótese que «Public Health», en la edición de 1946, se tradujo por «Sanidad Pública» en lugar de «Salud Pública». Véase una síntesis de la evolución de este concepto durante el franquismo en Pedro Marset, José M. Sáez, y Fernando Martínez-Navarro, «La Salud Pública durante el franquismo», Dynamis 15 (1995): 211-250.
} 
medicina como una ciencia social, sin rechazar su vinculación a las ciencias exactas. Esto último no implicaba que el médico olvidase que su «objeto de estudio, el "hombre", es un ser social, una unidad en una familia y un miembro de una comunidad».100 El sumario del libro muestra diferencias muy sustanciales con los manuales de higiene y medicina preventiva ya citados y publicados entre 1943, con las Lecciones del curso de Higiene de Zapatero, y 1967, con la tercera edición del libro de Piédrola, Pumarola y Bravo. ${ }^{101}$ La primera sección del libro de Smillie define conceptos e incluye demografía y estadística; la segunda remite al medio ambiente; la tercera al control de las enfermedades contagiosas; la cuarta, a la Higiene infantil; la quinta la dedica a las enfermedades crónico-degenerativas y a la salud mental; y, en la última, profundiza en la administración de la higiene social, describiendo los cambios en la administración sanitaria norteamericana desde antes del New Deal. Aunque este libro fue objeto de una recensión, ${ }^{102}$ no hemos podido documentar su influencia en España y, por consiguiente, debe de entenderse como una oportunidad perdida para introducir las tendencias internacionales sobre estas materias en España.

En 1951, el catedrático Valentín Matilla publicó un manual ${ }^{103}$ en cuyo prólogo se afirmaba que:

Si se quiere que el avance sanitario del país sea una realidad [...] se hace preciso que la masa ciudadana aprenda y asimile las doctrinas que la Higiene enseña y los principios sobre los que la Sanidad se asienta. Y para llegar a esta eficaz encarnación se precisa $[\ldots]$ que nuestras clases profesionales más directamente interesadas en esta labor posean una básica instrucción. ${ }^{104}$

Sin embargo, este nuevo manual siguió la estela de los de Zapatero y, aunque el segundo volumen contiene algo de legislación, asistencia

\footnotetext{
100 Smillie, Medicina preventiva, 17.

101 Gonzalo Piédrola, Agustín Pumarola y José Bravo, Higiene, medicina preventiva y social (Madrid: Amaro, 1967 [3. ${ }^{\mathrm{a}}$ ed.]).

102 Manuel Bermúdez, "Reseña de Smillie: "Medicina Preventiva y Sanidad Pública” , Revista de Sanidad e Higiene Pública 25 (1950): 390-391.

103 Valentín Matilla, Tratado de Higiene y Sanidad (Valencia: Saber, 1951).

104 Matilla, Tratado de Higiene, 7.
} 
pública e higiene colonial, carece de la amplitud temática del libro de Smillie.

\section{QUÉ MEDICINA SOCIAL PARA QUÉ CARRERA (1951-1959)}

La medicina social, como disciplina científica que requiere métodos y técnicas particulares, es muy anterior a la creación de los problemas prácticos que determinan su inclusión diferenciada en los planes de enseñanza universitaria. ${ }^{105}$

En 1951, Franco nombró a Joaquín Ruiz Giménez Ministro de Educación y éste promovió a Laín como Rector de la Universidad Central. Ambos mandatos se consideran un punto de inflexión en la evolución ideológica del régimen destinados a maquillar la «fascistización» y a permitir, entre otras acciones, el acceso a organismos internacionales como la OMS. ${ }^{106}$ Ello tuvo efectos en distintas esferas, también en educación y formación.

La progresiva implantación del SOE dio lugar a cambios en la relación médico-paciente. Algunos médicos generales, ya fueran de "zona» o rurales, reflexionaron - como veremos a continuación- acerca de estos nuevos problemas en su práctica profesional. En 1951, un editorial de la revista Horizonte ${ }^{107}$ afirmaba que:

$\mathrm{El}$ «problema social» $[\ldots]$ es una reacción lógica y biológica de la masa humana del mundo ante hechos pretéritos y presentes. [...] Es nuestro deseo entrar en el estudio de esos problemas de sociología para los cuales se encuentran movilizadas las inteligencias más preclaras de las que vamos recogiendo enseñanzas provechosas. ${ }^{108}$

En Horizonte, "problemas de sociología» debe entenderse como la problemática social derivada de la relación de los médicos con los

\footnotetext{
105 Primitivo de la Quintana, «La Medicina social», 110.

106 Esteban Rodríguez-Ocaña, «España y la OMS en tiempos de Palanca», Asclepio 71, n. 1 (2019), 254-265.

107 Horizonte fue una revista editada, entre 1949 y 1955, por la Comisión de Asesoramiento para el Seguro Obligatorio de Enfermedad (CASOE) del Colegio de Médicos de Barcelona.

${ }_{108}$ Sin autor, «Editorial: Medicina social, sí», Horizonte 3, n. 23 (1951): 1-3 pág. 1.
} 
beneficiarios del SOE. La conducta de los pacientes no se ajustaba a lo que esperaban los facultativos y, por eso, debían gestionar el conflicto mediante habilidades, aprendidas por su experiencia, que no se aprendían en la carrera. ${ }^{109} \mathrm{El}$ editorialista enlazaba la idea de que el problema social «tiene que ver con el arte de curar»,110 avalando la bondad de los contenidos del manual de Smillie.

\section{La reforma del plan de estudios de 1953}

En 1952,111 el Ministerio de Educación — tratando de reformar el plan de 1944- envió a Primitivo de la Quintana a explorar la situación de la medicina social y de las demás ciencias sociosanitarias en la formación médica internacional. El informe no se publicó en una revista sanitaria, sino en dos artículos de la Revista de Educación del Ministerio de Educación. En el primero, Quintana explica la anomalía que suponía la ausencia de medicina social en la formación médica. ${ }^{12}$ En el segundo se incidía en aspectos conceptuales y se describía la situación internacional. ${ }^{113}$ Denunciaba que:

En ninguna de las disciplinas que forman el plan de estudios de la carrera de Medicina se halla incluido un estudio, siquiera sea somero, de los fenómenos sociales más importantes, ni al estudiante se le suministran una serie de nociones que luego va a necesitar para comprender y valorar, en el transcurso de su vida profesional, la alusión permanente a estos fenómenos y a la configuración del medio donde debe actuar y donde se encuentra inmerso su paciente. ${ }^{114}$

Según Quintana, esa anomalía era fruto de las resistencias amparadas por el «reglamentismo» de algunos catedráticos «que no siempre

\footnotetext{
109 Perdiguero-Gil y Bueno, «Hay una diferencia entre», 103-113.

110 Sin autor, «Editorial: Medicina», 2.

111 Orden de 20 de Septiembre de 1952, por la cual se aplica provisionalmente la reforma de planes docentes en el primer curso de los estudios de las Facultades de Medicina, Ciencias, Farmacia y Ciencias Políticas y Económicas. BOE de 21 de septiembre de 1952, 265, 4310.

112 Quintana, «La Medicina», 1-11.

113 Quintana, «La Medicina social», 110-123.

114 Quintana, «La Medicina», 4.
} 
han mirado a los problemas sociales de su tiempo con la sensibilidad a que obliga su alto magisterio». ${ }^{115}$ Quintana, que estuvo vinculado a la planificación técnica del SOE y a la ENS, pero no al mundo universitario, defendía que la medicina social fuese una disciplina central e independiente dentro del currículo médico. Influido por el estructural-funcionalismo socio-antropológico, ${ }^{116}$ criticaba que:

El temario de cuestiones que ha ocupado la actividad normal de estas cátedras no ha destacado suficientemente los problemas que la evolución del pensamiento y la realidad social han ido creando en tiempos sucesivos, debido a la forma tradicional en que esta enseñanza se ha venido dando y a la historia de los problemas que constituían su contenido en el momento de su auge. ${ }^{117}$

Según Quintana, el temario era un corsé que impedía que el futuro médico conociese algo «que no está en relación directa con el organismo humano sino a través de su medio social y de la organización social».118 Aceptar sus propuestas hubiese supuesto un punto de inflexión en la formación en medicina social, pero la reforma de 1953 no recogió ninguna de ellas. ${ }^{119}$ Se limitó a mantener un curso preparatorio de Ciencias y seis cursos más, con Psicología en tercero, Psiquiatría y Pediatría y Puericultura, en sexto, e Historia de la Medicina e Higiene y Sanidad, en séptimo. Además, los diferentes manuales ya mencionados de higiene y medicina preventiva, como el publicado por Matilla en 1951,120 continuaron siendo la referencia para aprobar la asignatura de Higiene y Sanidad, así como para preparar los temarios a diversas oposiciones.

\footnotetext{
115 Quintana, «La Medicina», 1.

116 Los planteamientos de Quintana estaban alejados de la concepción filosófica de la antropología médica de Laín. Primitivo de la Quintana, Sociedad, cambio social y problemas de salud (Madrid: Real Academia Nacional de Medicina, 1966); Primitivo de la Quintana, «Medicina Social, Sociología Médica y Sociologia de la medicina», en Historia Universal de La Medicina, ed. Pedro Laín Entralgo, vol. VII (Barcelona: Salvat, 1978).

117 Quintana, «La Medicina», 4.

118 Quintana, «La Medicina», 8.

119 Decreto de 11 de agosto de 1953 por el que se establen los planes de estudios de las Facultades de Filosofía y Letras, Ciencias, Derecho, Medicina, Veterinaria y Ciencias Políticas, Económicas y Comerciales. BOE de 29 de agosto de 1953, 241, 5185-5190.
}

120 Matilla, Tratado de Higiene. 
En 1955, Luís Nájera reclamaba una profunda reforma de la enseñanza de la Higiene, aunque limitada a las metodologías cuantitativas. ${ }^{121}$ Esta actitud de apertura a las corrientes internacionales se puede documentar también en la Revista de Sanidad e Higiene Pública mediante las reseñas de obras como la ya citada de Smillie o la de Brockington, ${ }^{122}$ titulada World Health. ${ }^{123}$ Estas aportaciones, junto a las de Primitivo de la Quintana, demuestran que hubo un grupo de expertos de alto nivel, no necesariamente ubicados en las universidades, con un conocimiento de las disciplinas sociosanitarias homologable al internacional, pero que no se reflejó en los manuales académicos hasta una década más tarde.124 Sin duda, el ingreso de España en la OMS permitió que la DGS y la ENS, mediante la participación en congresos y simposios, ${ }^{125}$ incorporaran más fácilmente las novedades del momento.

\section{La tímida presencia de la sociología y la antropología}

En España, durante el franquismo, hablar de sociología o de antropología poco tenía que ver con el concepto actual de disciplina académica. Además, cuando se hablaba de antropología médica se hacía referencia a la propuesta de Laín.

El desarrollo académico de la sociología empírica se produjo, en los sesenta, sostenida por un mercado de trabajo específico como, entre otros, los informes de la Fundación FOESSA. ${ }^{126}$ En cambio, el caso de la antropología social y cultural fue más discreto, destacando el antropólogo

${ }^{121}$ Luís Nájera, «Sobre la reforma de la enseñanza de la higiene y medicina preventiva en las Facultades de Medicina», Revista de Sanidad e Higiene Pública 29, n. o 3-4 (1955): 171-187.

122 Sobre este autor, véase Esteban Rodríguez-Ocaña y Rosa Ballester, «El Informe (1967) del consultor de la OMS Fraser Brockington en el contexto del reformismo sanitario franquista», Dynamis 39, n. 2 (2019): 477-496

${ }^{123}$ Libro reseñado, en 1959, en la «Revista de Sanidad e Higiene Pública» (vol. 33: 385-387).

124 Enrique Nájera, «Estudio comparativo de la enseñanza de la Higiene y Sanidad en España y otros países», Revista de Sanidad e Higiene Pública 36, (1962): 279-325.

125 Por ejemplo, la participación en la Conferencia europea de estudios sobre la enseñanza post-universitaria de la Higiene, de la Medicina Preventiva y de la Medicina Social (Nancy, 1952); en la de profesores de Escuelas de Sanidad sobre enseñanza posgraduada de Medicina Preventiva y Social (Göteborg, 1953); o en la de la enseñanza de la Higiene, la Medicina Preventiva y la Medicina Social (Zagreb, 1956).

${ }^{126}$ Enrique Perdiguero-Gil y Josep M Comelles, «The Defence of Health. The Debates on Health Reform in 1970s Spain», Dynamis 39, n. 1 (2019): 45-72. 
Claudio Esteva y el pedagogo Adolfo Maíllo. El primero, en 1953, publicó un artículo sobre el papel social de la antropología aplicada en cultura, sanidad y educación, así como su experiencia como docente en la facultad de medicina durante su exilio en México. ${ }^{127}$ Vuelto a España, publicó dos artículos más en los que presentaba la teoría y métodos de la antropología social y cultural. ${ }^{128}$ Esta trilogía se publicó en revistas de primer nivel del periodo promovidas, en su mayoría, por Laín. Por su parte, Adolfo Maíllo, un técnico relevante del Ministerio de Educación, ${ }^{129}$ vindicó la necesidad de la institucionalización académica de la antropología y su papel en la educación sanitaria. ${ }^{130}$ Tanto Esteva como Maíllo reclamaron la antropología social y cultural aplicada a la intervención social.

Mientras, el médico y folklorista Antonio Castillo de Lucas publicó un manual, titulado Folkmedicina, ${ }^{131}$ destinado a estudiantes de medicina que continuaba remitiendo a un folklore médico ya en crisis. ${ }^{132}$ Introdujo el concepto de "etnomedicina», pero no desde la perspectiva relativista de la antropología social, sino considerando esos saberes populares como una realidad a aculturar bajo la teoría decimonónica del survivalism. ${ }^{133}$ En el prólogo, Laín — que no abdicaba de sus principios filosóficos-, distinguía tres etapas evolutivas en relación a la antropología: la etnográfica, histórica y la antropológica, que «consiste en interpretar y comprender que dentro de la naturaleza y la vida del hombre tiene la costumbre estudiada». ${ }^{134}$ Este modesto panorama se explica por la nula presencia académica de ambas disciplinas, cultivadas marginalmente en otras facultades y no en Medicina.

127 Claudio Esteva, «Panorama de la antropología mexicana», Cuadernos Hispanoamericanos 14, n. 37 (1953), 1-14.

128 Claudio Esteva, «La antropología contemporánea», Revista de Estudios Políticos 91 (1957): 95126; Claudio Esteva, «Sobre la teoría y los métodos de la antropología social», Revista Internacional de Sociología 15, n. 59 (1957): 411-436.

129 Juan Mainer y Julio Mateos, Saber, poder y servicio. Un pedagogo orgánico del Estado: Adolfo Maíllo (Valencia: Tirant Editorial, 2011).

${ }_{130}$ Adolfo Maíllo, «Los problemas de la educación popular», Revista de Educación 5, n. 12 (1953): 11-16.

${ }_{131}$ Antonio Castillo de Lucas, Folkmedicina: medicina popular, folklore médico, etnomedicina, demoiatria etnoiátrica (Barcelona: Dossat, 1958).

132 Enrique Perdiguero-Gil y Josep M. Comelles, «Medicina popular, compromiso profesional y educación sanitaria en España», Scripta Ethnologica 36 (2014): 7-40.

${ }^{133}$ Edward B. Tylor, Cultura primitiva, (Madrid, Ayuso, 1977).

${ }_{134}$ Castillo de Lucas, Folkmedicina, 8. 


\section{Qué formación para qué seguro}

En 1958, el catedrático Alfonso de la Fuente Chaos, uno de los ideólogos del SOE, publicó una suerte de libro blanco sobre el futuro de la medicina en España. ${ }^{135}$ Según él:

Las facultades de medicina no han cumplido en los últimos cincuenta años su misión institucional. Cada catedrático daba término a sus específicas enseñanzas con poco conocimiento de lo que hacía el resto. [...] Tampoco se preocuparon de explicar en cursos de postgraduados, la evolución de la Medicina, aun sabiendo los cambios profundos que habían hecho perder vigencia a las enseñanzas que proporcionaron. ${ }^{136}$

Aunque consideraba que la misión de la carrera era «enseñarle al estudiante que el español es un modo de ser ante la vida entera y ante la muerte» ${ }^{137}$ sus propuestas de reorganización del currículo médico privilegiaron una vez más las materias clínicas. Ante la más que probable ampliación de la cobertura del SOE y la reforma de los hospitales, era lógico pensar que las nuevas necesidades de los profesionales médicos pasaran por la medicina hospitalaria y de especialidades.

En 1959, solo un 40\% de la población española estaba cubierta por SOE, pero ya se observaban cambios en su cultura sanitaria. ${ }^{138}$ La gratuidad de la prestación farmacéutica del SOE había contribuido a que el binomio visita/receta fuese inseparable y a minimizar el papel de la educación sanitaria y de la prevención de la enfermedad. ${ }^{139}$ La Fuente Chaos era consciente de que la ampliación inminente de la cobertura sanitaria del SOE al medio rural conduciría a un aumento de la demanda de médicos y de especialistas e, inevitablemente, a una masificación de la carrera de medicina, como se puede comprobar ya en la primera mitad de los sesenta. Sin embargo, la reforma del plan de estudios de 1959 no amplió el número de facultades ni de plazas docentes. Mantuvo la posibilidad de

\footnotetext{
135 Alfonso de la Fuente-Chaos, Los grandes problemas de la medicina actual. Reforma de la enseñanza médica, plétora profesional, socialización de la Medicina (Barcelona: Editorial Científico-Médica, 1958).

136 Fuente-Chaos, Los grandes problemas, 28.

137 Fuente-Chaos, Los grandes problemas, 42.

138 Comelles et al., "Por caminos y veredas», 97.

139 Perdiguero-Gil y Comelles, «The Defence of Health», 58-60.
} 
la matrícula libre y se limitó a eliminar el curso preparatorio, que se trasladó al séptimo curso preuniversitario del Bachillerato, convirtiendo el primer curso de medicina en selectivo. ${ }^{140}$ No hubo cambios sustanciales en las materias. Para muchos catedráticos, cooptados tras la Guerra Civil, la perspectiva de su próxima jubilación no fue nunca un incentivo para una reforma a fondo ni, tampoco, el que no hubiese todavía grandes cambios en el mercado médico.

Los movimientos estudiantiles de 1956 no influyeron en la reforma de 1959. Esta debe relacionarse con la necesidad de atender a un previsible aumento de la demanda universitaria. Sin embargo, las resistencias internas y la «fosilización» de la Ley de 1943 impidieron responder a la masificación de la Universidad en la década siguiente, influyendo en su conflictividad interna. Fue inevitable crear nuevas universidades, en 1968, para ampliar una oferta académica casi inamovible desde finales del siglo XIX. ${ }^{141}$ Ante el crecimiento del mercado médico vinculado a la expansión de la Seguridad Social, a la reforma de los hospitales y al desarrollo del especialismo médico, no hubo más remedio que crear nuevas Facultades de Medicina para responder al desajuste entre la demanda de estudiantes y la capacidad docente de los centros. ${ }^{142}$

\section{CONCLUSIONES}

Estas consideraciones generales que venimos desarrollando en apoyo de la necesidad de establecer la Medicina social como disciplina autónoma en nuestras Facultades de Medicina, no han penetrado en ningún momento en el interés puramente científico de esta rama del saber. ${ }^{143}$

\footnotetext{
${ }^{140}$ Los alumnos no podían matricularse al segundo curso de la Licenciatura sin haber superado las pruebas de selección del primero. Decreto 1742/1959, de 23 de septiembre, por el que se reduce el plan de estudios del periodo de Licenciatura de la Facultad de Medicina. BOE de 12 octubre de 1959, 244, 13132.

${ }^{141}$ Sobre los cambios de la universidad española durante esta etapa, véase: Carlos París, «La Pretensión de Una Universidad Tecnocrática: panorama de la universidad española desde 1956 hasta 1975», en Carreras y Ruiz, La universidad española, 437-454.

142 Juan D. Tutosaus, Jesús Morán-Barrios y Fernando Pérez-Iglesias, «History of medical specialty training system in Spain and its teaching milestones», Educación Medica 19, n. ${ }^{\circ}$ (2018): 229-234.

143 Quintana, «La Medicina», 10.
} 
Este fragmento de 1953 podía haberse escrito en 1977, cuando se generalizó el programa de formación MIR (Médicos Internos y Residentes) en las Instituciones de la Seguridad Social. Su autor, Quintana, ya proponía incorporar tendencias internacionales que abogaban por una medicina social y una educación sanitaria novedosas. Visto el grado de conocimiento de la cuestión por parte de algunos técnicos de la DGS y de la ENS, debemos plantearnos por qué, más allá de un uso cosmético, no se llegaron a integrar estos conocimientos en la carrera de medicina. Podemos pensar que no fueron consecuentes en la aplicación de dicho conocimiento o que, por ser técnicos y no académicos, toparon con resistencias ideológicas al estar ubicados fuera de la Universidad. Lo cierto es que el valor marginal que las políticas de salud pública y educación sanitaria tuvieron para el régimen franquista, con la salvedad del modelo asistencial del SOE, les dejaron como voces aisladas y sin demasiada influencia. Prueba de ello es que, hasta la tercera edición del manual de Higiene y Medicina Preventiva y Social de Piédrola, Pumarola y Bravo de 1967,144 la estructura de esos manuales apenas cambió. A diferencia de los anteriores, este sí recogió algunas influencias internacionales que abogaban por la inclusión y potenciación de las ciencias sociosanitarias. ${ }^{145}$

A escala internacional, la formación médica de vanguardia había ido incorporando la medicina social y las demás ciencias sociosanitarias tanto en el pre como en el postgrado. Ello se consolidó, en 1978, cuando la OMS recomendó la implantación de la Atención Primaria de Salud, especialmente, dónde el proceso de medicalización era más lento —en particular el mundo poscolonial y rural- En España el primero era ya irrelevante, pero el segundo se vio agravado por el despoblamiento rural fruto de las migraciones masivas hacia las ciudades. Esto es, a las dificultades de acceso a los servicios asistenciales de la población rural, se añadieron los problemas sociales asociados a un crecimiento exponencial de la población

\footnotetext{
144 Piédrola, Pumarola y Bravo, Higiene (3. ${ }^{\mathrm{a}}$ ed.).

145 En la introducción de la edición de 1967, se recoge que en la XIX Asamblea Mundial de la Salud de Ginebra (mayo 1966), «al estudiarse las normas mínimas internacionales para la formación de Médicos, se ha insistido en la creciente importancia de la Medicina Preventiva [...] fijando firmemente la idea de que prevenir no solo significa evitar la enfermedad, sino también hacer al sano aún más sano». [Piédrola, Pumarola y Bravo, Higiene (3. ${ }^{\mathrm{a}}$ ed.), 20]. Ese mismo año, se publicó en España la traducción del libro de Mervyn W. Susser y William Watson, Sociology in Medicine (London: Oxford University Press, 1963). Sin embargo, este libro ya había sido reseñado tres años antes por Luis López-Villalba «Reseña del libro Sociology in Medicine», Revista de Sanidad e Higiene Pública 38 (1964): 223-224.
} 
urbana y de afiliación masiva al SOE. Además, el nuevo significado cultural de la atención especializada y hospitalaria subvirtió el tradicional modelo de relación médico-enfermo.

Por todas estas razones, en España, la formación médica tendría que haber incorporado, siguiendo la estela internacional, la enseñanza de la medicina social. Sin embargo, la presencia de contenidos sociosanitarios en la carrera de medicina siempre fue más bien escasa. Varias razones lo explican. Desde el «reglamentismo» que fosilizaba el contenido de las asignaturas y de los temarios de oposición hasta los obstáculos ideológicos y corporativos fruto de una organización de las facultades muy poco abierta a la interdisciplinariedad, pasando por razones político-ideológicas que retrasaron, hasta la Transición democrática, la evolución de la sanidad española hacia el concepto de salud pública.

\section{Nota sobre los autores}

Josep Barceló-Prats es actualmente profesor lector Serra Húnter en la Facultad de Enfermería de la Universitat Rovira i Virgili de Tarragona. También es miembro del grupo de investigación consolidado SGR380 «Enfermería Avanzada» y del Medical Anthropology Research Center de la mencionada universidad tarraconense. Doctor en Antropología, su principal línea de investigación se ha centrado en la historia de las instituciones asistenciales en España. En relación con esta materia ha sido galardonado, en 2014, con el XLV Premio de Historia de la Medicina de Fundación Uriach y, en 2019, con el XXIII Premio de Historia de la Medicina Catalana «Oleguer Miró i Borràs». En los últimos años, su actividad investigadora se ha ampliado con trabajos relacionados con la historia de las profesiones sanitarias y con el proceso de reforma hospitalaria durante el franquismo. Entre sus contribuciones recientes destacan: «Historical roots of hospital centrism in Catalonia (19171980)». Journal of Evolutionary Studies in Business 6, n. 1 (2021): 156181; L'evolució del dispositiu hospitalari a Catalunya (1849-1980). Manresa: PAHCS, 2020; «Las bases ideológicas y prácticas del proceso de regionalización de la sanidad en España (1955-1978)». En Salud, enfermedad y medicina en el franquismo, editado por M. Isabel Porras, Lourdes Mariño y M. Victoria Caballero, 146-167. Madrid: Los Libros de la Catarata, 2019. 
Josep M. Comelles es catedrático emérito en la Universitat Rovira i Virgili de Tarragona. Doctor en Medicina por la Universitat de Barcelona y en Antropología por la Ecole des Hautes Études en Sciences Sociales de París, es miembro fundador del Medical Anthropology Research Center de la citada universidad, sus campos de especialidad son la antropología médica y la historia de la ciencia (antropología, medicina y psiquiatría), la etnografía de la práctica profesional en salud y también los trastornos del comportamiento alimentario. En la última década ha desarrollado una línea de investigación sobre los cambios y los efectos colaterales derivados del proceso de medicalización en las sociedades occidentales y la progresiva reconversión de las agendas y del rol de los profesionales de la salud. Entre sus contribuciones recientes destacan: «De la descoordinación a la descentralización. La evolución del dispositivo hospitalario catalán durante el franquismo y la Transición (19391980)». En El Estado del bienestar entre el franquismo y la Transición, editado por Damián González-Madrid y Manuel Ortiz Heras, 231-259. Madrid: Sílex Editorial, 2020; «Fonts documentals per a l'estudi de la medicina rural durant el franquisme». Gimbernat: Revista d'Història de la Medicina $i$ de les Ciències de la Salut 73 (2020): 133-151; "The defence of health: the debates on health reform in 1970s Spain». Dynamis 39, n. 1 (2019): 45-72.

\section{REFERENCIAS}

Alted-Vigil, Alicia. «Bases político-ideológicas y jurídicas de la universidad franquista durante los Ministerios de Sainz Rodríguez y primera época de Ibáñez Martín (1938-1945)». En La universidad española bajo el régimen de Franco, editado por Juan J. Carreras y Miguel Á. Ruiz, 95-124. Zaragoza: Institución Fernando el Católico, 1991.

Báguena, M. ${ }^{a}$ José. "La higiene y la salud pública en el marco universitario español». Revista de Sanidad e Higiene Pública 68 (1994): 91-96.

Bonner, Thomas N. Becoming a Physician. Medical Education in Britain, France, Germany, and the United States, 1750-1945. New York: Oxford University Press, 1995.

Campos, Joan, y Jesús de Miguel. «Sociólogos de bata blanca y médicos con barba: Relaciones entre las ciencias sociales y la Medicina en España». Revista Española de Investigaciones Sociológicas 15 (1981): 133-144. 
Campos, Ricardo, y Ángel González. «Psiquiatría en el primer franquismo: saberes y prácticas para un "Nuevo Estado"». Dynamis 37, n. 1 (2017): $13-21$

Campos, Ricardo, y Enric Novella. «La Higiene Mental durante el primer franquismo. De la higiene racial a la prevención de la enfermedad mental (19391960)». Dynamis 37, n. 1 (2017): 65-87.

Casco-Solís, Juan. «Las topografias médicas: Revisión y cronología». Asclepio 53, n. ${ }^{\circ} 1$ (2001): 213-244.

Comelles, Josep M. «From Ethnography to Clinical Practice in the Construction of the Contemporary State». En Democracy and Ethnography: Constructing Identities in Multicultural Liberal States, editado por Carol J. Greenhouse, 233-253. Albany: State University of New York Press, 1998.

Comelles, Josep M. «The Role of Local Knowledge in Medical Practice: A Trans-Historical Perspective». Culture, Medicine and Psychiatry 24, n. 1 (2000): 41-75.

Comelles, Josep M., y Ángel Martínez-Hernáez. Enfermedad, cultura y sociedad. Un ensayo sobre las relaciones entre la Antropología social y la Medicina. Madrid: EUDEMA, 1993.

Comelles, Josep M., Enrique Perdiguero-Gil, Eduardo Bueno, y Josep Barceló-Prats. «Por caminos y veredas: la práctica médica rural bajo el franquismo (1939-1979)». En Genealogías de la reforma sanitaria en España, editado por José Martínez-Pérez y Enrique Perdiguero-Gil, 63-124. Madrid: Los Libros de la Catarata, 2020.

Danón, José (coord.). La Enseñanza de La Medicina en la universidad española: primera parte. Barcelona: Fundación Uriach, 1998.

Diasio, Nicoletta. La science impure. Anthropologie et médecine en France, Grande-Bretagne, Italie, Pays-Bas. Paris: PUF, 1999.

Foucault, Michael. El nacimiento de la clínica: una arqueología de la mirada médica. México: Siglo XXI editores, 2001.

González de Pablo, Ángel. «Por la psicopatología hacia Dios: Psiquiatría y saber de salvación durante el primer franquismo». Dynamis 37, n. ${ }^{\circ}$ (2017): 45-64. Jones, Colin. "The construction of the Hospital Patient in Early Modern France». En Institutions of Confinement. Hospitals, Asylums and Prisions in Western Europe and North America, editado por Norbert Finzsch y Robert Jütte, 55-74. Cambridge: Cambridge University Press, 1996.

Levy, Silvia. Psicoanálisis y defensa social en España, 1923-1959. Madrid: Los Libros de la Catarata, 2019.

López-Piñero, José M. «La enseñanza médica en España desde la Baja Edad Media hasta la Ley Moyano (1857)». En La enseñanza de la Medicina en la universidad española: primera parte, coordinado por José Danón, 7-29. Barcelona: Fundación Uriach, 1998. 
López Sánchez, José M. "La Medicina en la universidad nacionalcatólica». En La universidad nacionalcatólica. La reacción antimoderna, editado por Luis E. Otero, 317-476. Madrid: Universidad Carlos III, 2014.

Mainer, Juan, y Julio Mateos. Saber, poder y servicio. Un pedagogo orgánico del Estado: Adolfo Maíllo. Valencia: Tirant Editorial, 2011.

Marset, Pedro, José M. Sáez, y Fernando Martínez-Navarro. «La Salud Pública durante el franquismo». Dynamis 15 (1995): 211-250.

Martínez-Hernáez, Ángel, Enrique Perdiguero-Gil, y Josep M. Comelles. «Genealogía de la Antropología médica en España». Revista de Dialectología y Tradiciones Populares 70 (2015): 205-233.

Martínez-Navarro, Ferran. «La Sanidad en España». Revista de Sanidad e Higiene Pública 51 (1977): 777-817.

Menéndez, Eduardo L. «El modelo médico y la salud de los trabajadores». Salud Colectiva 1, n. ${ }^{\circ} 1$ (2005): 9-32.

Miguel, Jesús M. de. «Fundamentos de sociología de la medicina». Papers: Revista de Sociología 5 (1976): 209-239.

Molero, Jorge, e Isabel Jiménez. «Salud y burocracia en España. Los Cuerpos de Sanidad Nacional, 1855-1951». Revista Española de Salud Pública 74 (2000): 45-79.

Otero, Luis E. «La universidad nacionalcatólica». En La Universidad Nacionalcatólica. La reacción antimoderna, editado por Luis E. Otero, 69-130. Madrid: Universidad Carlos III, 2014.

París, Carlos. «La pretensión de una Universidad tecnocrática (panorama de la Universidad española desde 1956 hasta 1975)». En La Universidad Española Bajo El Regimen de Franco (1939-1975), editado por Juan J. Carreras y Miguel A. Ruiz, 437-454. Zaragoza: Institución Fernando el Católico, 1991.

Perdiguero, Enrique, y Josep M. Comelles. «Medicina popular, compromiso profesional y educación sanitaria en España». Scripta Ethnologica XXXVI (2014): 7-40.

Perdiguero-Gil, Enrique. «La «educación sanitaria» en el ideario médico-social del franquismo a través de la revista SER». En Política, salud y enfermedad en España: entre el desarrollismo y la transición democrática, editado por Enrique Perdiguero-Gil. Elche: Universidad Miguel Hernández, 2015.

Perdiguero-Gil, Enrique, Josep M. Comelles, y Aida Terrón. «La introducción de las ciencias sociomédicas en el ámbito de la educación sanitaria y la educación médica durante el franquismo». En Al Servicio de la salud humana. La historia de la medicina ante los retos del Siglo XX, editado por Alfons Zarzoso y Jon Arrizabalaga, 123-128. Sant Feliu de Guíxols: SEHM, 2017.

Perdiguero-Gil, Enrique, y Josep M Comelles. «The Defence of Health. The Debates on Health Reform in 1970s Spain». Dynamis 39, n. 1 (2019): 45-72. 
Perdiguero-Gil, Enrique, y Eduardo Bueno. «Hay una diferencia entre la medicina social y la socializada. Las resistencias de los médicos españoles a la colectivización de la asistencia sanitaria y la ampliación de la cobertura sanitaria (1944-1963)». En El Estado del bienestar entre el franquismo y la Transición, editado por Damián González-Madrid y Manuel Ortíz, 95-124. Madrid: Silex Editorial, 2020.

Pérez López-Villamil, José. Manual de Psiquiatría. Psicopatología General. Lugo: Asociación Gallega de Psiquiatria, 2003.

Peset, Mariano. «La Ley de Ordenación Universitaria de 1943». En La universidad española bajo el régimen de Franco, editado por Juan J. Carreras y Miguel Á. Ruiz-Carnicer, 125-158. Zaragoza: Institución Fernando el Católico, 1991.

Peset, Mariano. «Prólogo». En Oposiciones y concursos a cátedras de Historia en la Universidad de Franco (1939-1950), editado por Yolanda Blasco y María F. Mancebo, 11-22. València: Publicacions de la Universitat de València, 2010.

Peset, Mariano, y José L. Peset. La universidad española (siglos XVIII y XIX): despotismo ilustrado y revolución liberal. Madrid: Taurus, 1974.

Ridruejo, Alejandro. «La Enseñanza de la medicina en España: planes de estudio (1843-1931)». Tesis doctoral, Universidad de Valladolid, 1979.

Rodríguez Ocaña, Esteban. La constitución de la medicina social como disciplina en España (1882-1923). Madrid: Ministerio de Sanidad y Consumo, 1987.

Rodríguez-Ocaña, Esteban. «La intervención de la Fundación Rockefeller en la creación de la sanidad contemporánea en España». Revista Española de Salud Pública 74 (2000): 27-34.

Rodríguez Ocaña, Esteban. «Ocaso de la medicina social en España: El caso de la Leptospirosis», Asclepio 69, n. 2 (2017): 1-12.

Rodríguez Ocaña, Esteban. «España y la OMS en tiempos de Palanca». Asclepio 71, n. 1 (2019): 254-265.

Rodríguez-Ocaña, Esteban y Rosa Ballester. «El Informe (1967) del consultor de la OMS Fraser Brockington en el contexto del reformismo sanitario franquista». Dynamis 39, n. 2 (2019): 477-496.

Rosen, George. De la policía médica a la medicina social: ensayos sobre la historia de la atención a la salud. México: Siglo XXI, 1985.

Ruiz Falcó, Fernando. «Escuela Nacional de Sanidad. Problemática pasada, actual y futura». Revista de Sanidad e Higiene Pública 57 (1983): 359-372.

Salazar, Modesta, Josep Bernabeu-Mestre, y Emilio A. Martínez-Marco. «La salud materno-infantil durante el franquismo: notas bibliométricas sobre el programa “Al Servicio de España y del niño español”». Asclepio 59, n. ${ }^{\circ} 1$ (2007): 285-314.

Serrallonga, Joan. "Reformadores y reaccionarios en la estructura central de sanidad en España, 1931-1936». Investigaciones Históricas 29 (2009): 241264. 
Sesma, Nicolás. "Sociología del Instituto de Estudios Políticos. Un "grupo de élite" intelectual al servicio del Partido Único y del Estado franquista». En Falange: las culturas políticas del fascismo en la España de Franco (19361975), editado por Miguel Á. Ruiz-Carnicer, vol. 1, 253-288. Zaragoza: Institución Fernando el Católico, 2013.

Stocking, George W. «Anthropology and the Science of the Irrational. Malinoswski's Encounter with Freudian Psychoanalysis». En Malinowski, Rivers, Benedict and Others. Essays on Culture and Personality, editado por George W. Stocking, 13-49. Madison: The University of Wisconsin Press, 1986.

Stocking, George W. «The Ethnographic Sensibility of the 1920s and the Dualism of the Anthropological Tradition». En Romantic Motives. Essays on Anthropological Sensibility, editado por George W. Stocking, 208-276. Madison: The University of Wisconsin Press, 1989.

Terrón, Aida, Josep M. Comelles, y Enrique Perdiguero-Gil. «Schools and Health Education in Spain during the Dictatorship of General Franco (19391975)». History of Education Review 46, n. ${ }^{\circ}$ (2017): 208-223.

Tutosaus, Juan D., Jesús Morán-Barrios y Fernando Pérez-Iglesias. «History of Medical Specialty Training System in Spain and its Teaching Milestones». Educación Medica 19, n. 4 (2018): 229-234.

Tylor, Edward B. Cultura primitiva. Madrid, Ayuso, 1977.

Zarzoso, Alfons, y Àlvar Martínez-Vidal, Medicina, guerra y exili. Una generació destruïda per la guerra. Barcelona: CSIC, 2011. 